\title{
Towards an Evolution of E-Learning Recommendation Systems: From 2000 to Nowadays
}

\author{
https://doi.org/10.3991/ijet.v16i06.18159 \\ Sonia Souabi ${ }^{(凶)}$, Asmaâ Retbi, Mohammed Khalidi Idrissi, Samir Bennani \\ Mohammed V University,Rabat, Morocco \\ souabisonialgmail.com
}

\begin{abstract}
In e-learning, recommendation systems have proven to be highly efficient for improving learners' performance and knowledge. They can manage the different pedagogical resources and simplify the workload for the instructor and learners as well. Throughout the years, recommendation systems in elearning have witnessed a major evolution since the 2000s. Several aspects have been developed, including techniques involved, test data (...). In this respect, this paper analyses the evolution of recommendation systems in e-learning since 2000 with a focus on the evolution sides. It furthermore addresses areas not fully addressed to date. A set of recommendation systems is identified and then analysed in order to define techniques used, as well as algorithms deployed.
\end{abstract}

Keywords - E-learning, recommendation systems, evolution.

\section{$1 \quad$ Introduction}

The large amount of information and material has hindered the task of learners as they are encountering many educational resources covering different subjects. Elearning improves learners' knowledge and abilities [1-2]. Mooc, in particular, offers several options for learners and a diversity of material [3-4]. Choosing between all the existing information and materials is currently regarded as one of the toughest choices requiring prior study and analysis of all features of the learning environment. In this respect, recommendation systems can manage multiple resources within a learning environment and assist in guiding learners towards a more efficient learning process [5-6]. Many researchers addressed recommendation systems in e-learning [79]. Techniques and approaches covered are also varied, from personalized approaches, collaborative filtering approaches to hybrid approaches [10-12]. Recommendation systems emerged early in research, although they did not start to grow in the field of e-learning until the 2000s. It is only from this year onwards that researchers began to explore the function of recommendation systems in e-learning and how they can guide learners in their learning. In e-learning, recommendation systems are progressing with the advancement of Machine Learning algorithms and Big Data techniques. For instance, learning analysis can be handled to predict learner performance within Moodle [13]. It is therefore of utmost importance to study the evolution of recommendation systems since their emergence. In this article, we propose a study analyzing the evolu- 
tion of recommendation systems in e-learning since 2000 and discussing the points developed by researchers according to their types and characteristics. On the other hand, we plan to explore limitations of studies already carried out in e-learning on recommendation systems and what are the next avenues to pursue.

The paper is structured according to the following parts: the first part deals with the research methodology in question, including data collection; the second part consists of an analysis of selected papers; the third part provides a general analysis of results obtained after selecting papers and thus gives answers to questions asked; finally, the conclusion elaborates results of the analysis and the next avenues to be explored.

\section{$2 \quad$ Analytical Technique}

\subsection{Research questions}

In this study, we will analyze the different selected researches in order to detect which techniques have been contemplated as well as areas not covered through the two questions:

a) What are challenges that have been addressed by recommendation systems?

b) What are the limits that were not included in these studies?

\section{$2.2 \quad$ Research methodology}

\section{- Data collection}

To select papers, we spread our study over four time periods:

a) From 2000 to 2005

b) From 2006 to 2010

c) From 2011 to 2015

d) From 2016 to 2020

Research was conducted on several databases, including IEEE, Springer, Elsevier, ScienceDirect, Google Scholar.

\section{- Search by keyword}

Research terms used for this article can be divided into two groups. The first group includes terms related to e-learning: e-learning, distance learning, learners, online education. The second group includes terms referring to recommendation systems and their different types: recommendation system, recommendation, content, collaborative filtering, hybrid system. 


\section{- Selection of papers to be included}

Papers selected must meet the following criteria:

a) They must be written in English

b) They must be published between 2000 and 2020

c) Two words should basically appear in the title: recommendation and e-learning

d) Focus on articles appearing at the beginning of the research list and with a high number of citations

\section{Analysis of the Selected Papers}

This part is devoted to the study of recommendation systems proposed in the context of e-learning and social learning. We will study the evolution of recommendation systems in this field from 2000 up to the present moment. We will thus look more closely at recent proposed recommendation systems and the aspects that have not been addressed in depth.

\subsection{First period: From 2000 to 2005}

Recommendation systems have coexisted since the 1990s. It was only after the 1990s that recommendation systems started to spread in many areas, including elearning. As a result, recommendation systems within e-learning only began to gain prominence after 2000. In this period, researchers initiated their work in exploring these systems in e-learning and how these systems will improve the quality and performance of e-learning. We report an overview of some work undertaken in the area of e-learning recommendation systems.

\section{- A personalized e-learning recommender system [14]}

This study proposes a personalized recommendation system for two purposes: the first purpose is to identify and justify the needs of learners and the second purpose is to recommend content that is appropriate to the identified needs of learners. The framework consists of several parts:

a) Extraction of learners' data

b) Identification of learners' requirements and needs

c) Content analysis

d) Generation of recommendations

\section{- A social recommendation system [15]}

This article proposes a QSIA recommendation system aiming at sharing questions and knowledge among learners. The concept of social mechanisms is discussed while comparing the aspect of neighbours compared to friends. 


\section{- A recommender agent for e-learning systems [16]}

The concept of actions carried out by users has been widely adopted in several areas. This article focuses on techniques for exploiting the web based on learners' activities and their history. The approach consists in creating a recommendation agent based basically on association rules. The association rules thus focus on linking different items simultaneously.

\section{- A smart recommendation system [17]}

The study proposes a recommendation system adapted both to the different users and to the web environment as well. The system takes several features into consideration:

a) Basic contents

b) Learner evaluation

c) Collecting information on the web environment

d) Collaborative classification and filtering module

\section{- A personalised recommendation system [18]}

An EPERS recommendation system is designed to offer learners personalised content meeting their requirements. The framework is based on numerous aspects:

a) Extraction of learner-related information

b) Knowledge of the field

c) Learning resources for recommendations

\section{- An e-learning system based on item response theory [19]}

The recommendation system proposed in this article makes it possible to consider the degree of difficulty of pedagogical subjects as well as the capabilities of learners. The architecture of the recommendation system depends on several parameters:

a) Learner data

b) Data related to available educational resources

c) Explicit learner assessments

The recommendation system is thus evaluated by considering explicit feedback from learners.

A significant amount of Research has been accomplished on recommendation systems in e-learning between 2000 and 2005. Although e-learning recommendation systems only emerged in the 2000s, researchers have explored several avenues for personalized recommendations, social recommendations and explicit feedback from learners. More emphasis has been devoted to personalized recommendation systems to meet learners' requirements and needs. 


\subsection{Second period: From 2006 to 2010}

Recommendation systems are starting to gain prominence during this period. How then have recommendation systems evolved during these years, and what changes and improvements have researchers brought forward in comparison to what has previously been achieved? We outline some of the recommending systems proposed from 2006 to 2010 .

\section{- A recommendation system based on collaborative filtering [20]}

The proposed recommendation system is based mainly on the weight of learners according to their level of knowledge. Those with a higher level will have higher weights than those with a lower level. In this respect, equations have been modified in connection with collaborative filtering. The test was carried out on MovieLens as long as the e-learning realm suffers from a lack of data.

\section{- A recommender system based on implicit feedbacks [21]}

This article proposes to consider implicit feedback from learners instead of relying on explicit data and learner evaluations. This entails applying web data mining to build a better recommendation system. Association rules are proving to play a significant contribution to recommendations in the context of web mining.

\section{- A smart system on e-learning [22]}

A web application is developed integrating the recommendation system aiming to support the learning process for learners. This keeps essential questions and resources.

\section{- Predicting student performance by a recommender system [23]}

This approach uses recommendation systems to predict learner performance. The assessment was carried out on numerous databases, also based on linear regression and logistic regression.

\section{- A hybrid recommender system [24]}

A hybrid system is set up within a learning environment combining content-based and classification-based techniques. Classification algorithms such as k-means have been adopted to achieve adequate content clustering.

\section{- A hybrid system [25]}

This hybrid system proposes to combine concept maps and collaborative filtering to better identify the learning process. The system encompasses several parts:

a) The pedagogical resource base

b) The tag base

c) The base of concept maps 
During this phase, hybrid recommendation systems occupy an important position in the e-learning domain. Researchers are realizing the relevance of hybrid recommendation systems as they offer more advantages and performance compared to other recommendation systems.

\subsection{Third period: From 2011 to 2015}

This period is marked by the high prevalence of Machine Learning algorithms and more developed techniques as well as more reliable systems. What are the then the areas of improvement and development? We thus identify some studies performed on e-learning recommendation systems between 2011 and 2015.

\section{- Application of recommender systems on e-learning environments [26]}

The recommendation system proposed in this article consists in ensuring appropriate content for learners according to their level. The system is then founded on several aspects:

a) Recorded data about learners

b) Classification of learners with a higher level

c) Association rules for learners with a lower level

d) Recommendations according to the category of the learner

\section{- A context-based recommendation system [27]}

This study proposes the integration of learners' implicit activities as an alternative to explicit answers. The approach is based on two aspects:

a) The geographical context

b) The user context

\section{- Adaptive hypermedia for e-learning personalization [28]}

A developed program is suggested, Protus, which intends to combine recommendations and adaptive hypermedia. The architecture of protus consists of various parts:

a) The learner interfaces

b) The system server

c) The teacher interfaces

\section{- Semantic-based recommendation system [29]}

The recommendation approach proposed in this article sets up a system based on semantic analysis and the relationship between object learning and learners' needs. The architecture of the semantic recommendation system consists of:

a) Repository service

b) Indexing service

c) User service 


\section{- An e-learning recommender systems based on hybrid filtering [30]}

The approach comprises a two-sided hybrid recommendation system, the first side is a hybridisation between content filtering and collaborative filtering, while the second side is a hybridisation between collaborative filtering and the k-neighbour algorithm. The framework consists of several aspects:

a) Learner profile

b) Relevant contents

c) Recommendations addressed to the target learner

\section{- A hybrid system [31]}

This hybrid system consists on the one hand of collaborative resource filtering and then applying the sequential algorithm to detect common resources. The hybrid approach includes:

a) Web logs

b) Data pre-processing

c) Sequential models

d) Recommendation results

We distinguish a heavy presence of hybrid recommendation systems. Researchers are more interested in combining developed approaches and algorithms to increase the performance of their recommendation systems.

\subsection{Fourth period: From 2016 to 2020}

\section{- Ontology-based systems [32]}

This article presents a review of papers produced between 2005 and 2014 within the framework of ontology-based recommendation systems in e-learning. The study ranks the papers according to the type of ontology and the type of programming language.

- A hybrid system based on sequential pattern mining and context awareness [33]

This paper proposes a hybrid recommendation system combining the contextual aspect, sequential analysis and collaborative filtering algorithms. The hybrid approach covers:
a) Web logs
b) Learner profiles
c) The learning model 


\section{- A hybrid system based on ontology [34]}

A hybrid recommendation system is proposed combining ontology and sequential analysis. The approach consists of calculating similarity based on the ontology, then generating the top $\mathrm{N}$ recommendations, and finally applying the sequential analysis to generate the final recommendations.

\section{- A graph-based system [35]}

This recommendation system suggests the graphical study to generate recommendations. Several semantic relations have been explored between learning objects. The proposed recommendation structure includes the knowledge graph, the learning path and recommendations.

- A fog-based recommendation system [36]

The proposed recommendation system contains three modules:

a) Class identification module: calculating the correlation factor

b) Subclass identification module

c) Correspondence module: matching subclasses to available contents

\subsection{Interpretation of results}

To analyse results arising from our analysis based on work between 2000 and 2020, we will classify the different approaches into the following categories: Personnalized, collaborative filtering, hybrid, web logs and data, semantic and others. After sorting the research studied into these categories, we get the following graph (figure $1)$.



Fig. 1. Distribution of research carried out on e-learning recommendation systems between 2000 and 2020 
The graph reveals that hybrid recommendation systems have held a prominent position in e-learning recommendation systems for many years. This indicates that researchers tend to focus on hybrid approaches as they outperform traditional systems. On the other hand, web logs are also strongly present in e-learning recommendation systems since they allow tracking learner activity, and hence offer recommendations that are more appropriate to learners' profiles and to actions taken within the learning environment.

In the coming section, we will respond to the questions addressed at the beginning of the article.

\section{a) What challenges were addressed by the recommendation systems discussed?}

The recommendation systems cited covered a broad range of techniques:

i. Collaborative filtering: which is based on all content and data from different learners.

ii. Hybrid techniques: which are based on the combination of several techniques simultaneously to improve the performance of the recommendation system.

iii. Web logs: which are addressed in many studies so as to better respond to learner requirements.

iv. Ontology: which is considered among the most important aspects in recommendations.

v. Personalized recommendations: based on learner profiles and personalized data of each learner.

It also turns out that significant importance has been accorded to hybrid recommendation systems, as they can combine the advantages of several techniques and obtain more reliable results.

\section{b) What are the limits and points that have not been addressed in these studies?}

Although the systems of recommendations identified from 2000 to 2020 addressed many techniques, there are still several outstanding issues and aspects to be explored, as well as several challenges to be met:

i. Focusing more on exploring social distance learning: In fact, most studies address e-learning within the general context and do not specifically focus on social learning environments, including social networks. Social learning responds to specific needs and encourages interactivity and collaboration among learners.

ii. Lack of implicit data in web data mining: Analyzing web data is deemed more difficult compared to explicit learner data. In some instances, it is difficult to find all the data related to learners' logs in order to obtain relevant recommendations.

iii. In other cases, logs do not reflect the real preferences of learners, assuming that certain actions are not of considerable importance.

iv. The problem of Data Scarcity in e-learning has not been properly handled. Some research has used other databases from other fields to prove the performance of 
their recommendation systems. However, in order to evaluate a recommendation system in e-learning, the database must relate to the learning context.

v. Treating each action as independent of the other: In most cases, recommendation systems tend to consider actions undertaken by learners as independent when this is not necessarily the case. In this respect, it is important to measure the degree of correlation between the events carried out by learners and integrate this parameter in the calculation of relevant recommendations.

\section{Conclusion}

In the context of e-learning, recommendation systems are of utmost importance. They ensure a good management of pedagogical resources and time for learners, and offer a diversity of contents in order to improve the learning level of learners. Since 2000 , recommendation systems started to become popular in e-learning, including content-based systems, collaborative filtering and hybrid systems. To describe the evolution of recommendation systems in e-learning over the years, we split the research into four parts to analyze issues that were addressed in each period and changes and modifications proposed. In this article, we also highlight the aspects that have been considered by researchers since 2000 as well as challenges that have not been raised. Based on our study, we foresee in our future work:

i. To propose a recommendation system within the social learning perspective addressing the problem of data scarcity and implicit data gaps.

ii. Demonstrate the performance and reliability of our proposed recommendation system.

\section{$5 \quad$ References}

[1] A. Ewais and D. Abu Samara, « Adaptive MOOCs Based on Intended Learning Outcomes Using Naïve Bayesian Technique », Int. J. Emerg. Technol. Learn., vol. 15, no 04, p. 4, févr. 2020, https://doi.org/10.3991/ijet.v15i04.11420

[2] N. Ratnawati and I. Idris, "Improving Student Capabilities through Re-search-Based Learning Innovation on E-Learning System », Int. J. Emerg. Technol. Learn., vol. 15, no 04, p. 195, févr. 2020, https://doi.org/10.3991/ijet.v15i04.11820

[3] A. H. Albashtawi and K. B. Al Bataineh, "The Effectiveness of Google Classroom Among EFL Students in Jordan: An Innovative Teaching and Learning Online Platform », Int. J. Emerg. Technol. Learn., vol. 15, no 11, p. 78, juin 2020, https://doi.org/10.3991/ijet. v15i11.12865

[4] Y. Benjelloun Touimi, A. Hadioui, N. El Faddouli, and S. Bennani, « Intelligent ChatbotLDA Recommender System », Int. J. Emerg. Technol. Learn., vol. 15, no 20, p. 4, oct. 2020, https://doi.org/10.3991/ijet.v15i20.15657

[5] I. Mazeh and E. Shmueli, « A personal data store approach for recommend-er systems: enhancing privacy without sacrificing accuracy », Expert Systems with Applications, vol. 139, p. 112858, janv. 2020, https://doi.org/10.1016/j.eswa.2019.112858 
[6] Y. Pan, F. He, H. Yu., H. Li, « Learning adaptive trust strength with user roles of truster and trustee for trust-aware recommender systems », Appl Intell, vol. 50, no 2, p. 314-327, févr. 2020, https://doi.org/10.1007/s10489-019-01542-0

[7] K. Sandhu and H. Alharbi, « PLS Model Performance for Factors Influencing Student Acceptance of E-Learning Analytics Recommender »:, International Journal of Virtual and Personal Learning Environments, vol. 10, no 2, p. 1-14, juill. 2020, https://doi.org/10. 4018/ijvple.2020070101

[8] O. E. Aissaoui and L. Oughdir, « A learning style-based Ontology Matching to enhance learning resources recommendation », in 2020 1st International Conference on Innovative Research in Applied Science, Engineering and Technology (IRASET), Meknes, Morocco, avr. 2020, p. 1-7, https://doi.org/10.1109/iraset48871.2020.9092142

[9] Y. Zhang, A. Ghandour, and V. Shestak, « Using Learning Analytics to Predict Students Performance in Moodle LMS », Int. J. Emerg. Technol. Learn., vol. 15, no 20, p. 102, oct. 2020, https://doi.org/10.3991/ijet.v15i20.15915

[10] Mawane J., Naji A., Ramdani M. (2020) Unsupervised Deep Collaborative Filtering Recommender System for E-Learning Platforms. In: Hamlich M., Bel-latreche L., Mondal A., Ordonez C. (eds) Smart Applications and Data Analysis. SADASC 2020. Communications in Computer and Information Science, vol 1207. Springer, Cham. https://doi.org/ 10.1007/978-3-030-45183-7 11

[11] C. De Medio, C. Limongelli, F. Sciarrone, and M. Temperini, « MoodleREC: A recommendation system for creating courses using the moodle e-learning plat-form », Computers in Human Behavior, vol. 104, p. 106168, mars 2020, https://doi.org/10.1016/j.chb.2019. $\underline{106168}$

[12] J. K. Tarus, Z. Niu, and G. Mustafa, « Knowledge-based recommendation: a review of ontology-based recommender systems for e-learning ", Artif Intell Rev, vol. 50, no 1, p. 21-48, juin 2018, https://doi.org/10.1007/s10462-017-9539-5

[13] B. Abdelghani, « Trends in MOOCs Research: Analysis of Educational Technology Journals », Int. J. Emerg. Technol. Learn., vol. 15, no 17, p. 47, sept. 2020, https://doi. org/10.3991/ijet.v15i17.14637

[14] Jie Lu, « A Personalized e-Learning Material recommender system», Proceedings of the 2nd International Conference on Information Technology for Application (ICITA 2004).

[15] Sheizaf Rafaeli and Miri Barak, « Social recommender systems: recommendations in support e-learning», in International Journal of Distance Education Technologies (IJDET).

[16] O. R. Zaiane, " Building a recommender agent for e-learning systems », in International Conference on Computers in Education, 2002. Proceedings., Auckland, New Zealand, 2002, vol. 1, p. 55-59, https://doi.org/10.1109/cie.2002.1185862

[17] T. Y. Tang and G. Mccalla, « Smart Recommendation for an Evolving E-Learning System », p. 13.

[18] D. M. A. Otair, « Expert Personalized E-Learning Recommender System », p. 5.

[19] C.-M. Chen, H.-M. Lee, and Y.-H. Chen, « Personalized e-learning system us-ing Item Response Theory », Computers \& Education, vol. 44, no 3, p. 237-255, avr. 2005, https://doi.org/10.1016/j.compedu.2004.01.006

[20] J. Bobadilla, F. Serradilla, and A. Hernando, « Collaborative filtering adapted to recommender systems of e-learning », Knowledge-Based Systems, vol. 22, no 4, p. 261-265, mai 2009, https://doi.org/10.1016/j.knosys.2009.01.008

[21] O.R.Zainze, «Recommender systems for e-learning: towards non-intrusive web mining», Data mining in e-learning, p.80, 2006.

[22] Soonthornphisaj N., Rojsattarat E., Yim-ngam S. (2006) Smart E-Learning Using Recommender System. In: Huang DS., Li K., Irwin G.W. (eds) Computational Intelligence. ICIC 
2006. Lecture Notes in Computer Science, vol 4114. Springer, Berlin, Heidelberg. https://doi.org/10.1007/978-3-540-37275-2_63

[23] N. Thai-Nghe, L. Drumond, A. Krohn-Grimberghe, and L. Schmidt-Thieme, " Recommender system for predicting student performance », Procedia Computer Science, vol. 1, no 2, p. 2811-2819, 2010, https://doi.org/10.1016/j.procs.2010.08.006

[24] Leyla Zhuhadar and Olfa Nasraoui, « A Hybrid Recommender System Guided by Semantic User Profiles for Search in the E-learning Domain », Journal of Emerging Technologies in Web Intelligence, vol. 2, no. 4, November 2010. https://doi.org/10.4304/jetwi.2.4.272$\underline{281}$

[25] A. A. Kardan, S. Abbaspour, et F. Hendijanifard, « A Hybrid Recommender System for Elearning Environments Based on Concept Maps and Collaborative Tagging », p. 8, 2009.

[26] A. Sekhavatian and M. Mahdavi, "Application of Recommender Systems on e-learning Environment », p. 10.

[27] D. Gallego, E. Barra, P. Rodriguez, and G. Huecas, « Incorporating proactivity to contextaware recommender systems for e-learning ", in 2013 World Congress on Computer and Information Technology (WCCIT), Sousse, Tunisia, juin 2013, p. 1-6, https://doi.org/ $10.1109 /$ wccit.2013.6618700

[28] Boban Vesin, Aleksandra Kla`snja-Milicevi ', « Applying recommender systems and adaptive hypermedia for e-learning personnalization », Computing and Informatics, Vol. 00, 2012, 0-30, V 2011-Aug-24

[29] S. Fraihat and Q. Shambour, «A Framework of Semantic Recommender System for eLearning ", JSW, vol. 10, no 3, p. 317-330, mars 2015, https://doi.org/10.17706/ jsw.10.3.317-330

[30] M. W. Chughtai, A. Selamat, I. Ghani, and J. J. Jung, « E-Learning Recom-mender Systems Based on Goal-Based Hybrid Filtering », International Journal of Distributed Sensor Networks, vol. 10, no 7, p. 912130, juill. 2014, https://doi.org/10.1155/2014/912130.

[31] W. Chen, Z. Niu, X. Zhao, and Y. Li, « A hybrid recommendation algorithm adapted in elearning environments », World Wide Web, vol. 17, no 2, p. 271-284, mars 2014, https://doi. org/10.1007/s11280-012-0187-Z

[32] J. K. Tarus, Z. Niu, and G. Mustafa, « Knowledge-based recommendation: a review of ontology-based recommender systems for e-learning », Artif Intell Rev, vol. 50, no 1, p. 21-48, juin 2018, https://doi.org/10.1007/s10462-017-9539-5

[33] J. K. Tarus, Z. Niu, and D. Kalui, « A hybrid recommender system for e-learning based on context awareness and sequential pattern mining ", Soft Com-put, vol. 22, no 8, p. 2449-2461, avr. 2018, https://doi.org/10.1007/s00500-017-2720-6

[34] J. K. Tarus, Z. Niu, and A. Yousif, « A hybrid knowledge-based recommender system for e-learning based on ontology and sequential pattern mining », Future Generation Computer Systems, vol. 72, p. 37-48, juill. 2017, https://doi.org/10.1016/j.future.2017.02.049

[35] D. Shi, T. Wang, H. Xing, and H. Xu, « A learning path recommendation model based on a multidimensional knowledge graph framework for e-learning ", Knowledge-Based Systems, vol. 195, p. 105618, mai 2020, https://doi.org/10.1016/j.knosys.2020.105618

[36] T. S. Ibrahim, A. I. Saleh, N. Elgaml, and M. M. Abdelsalam, « A fog based recommendation system for promoting the performance of E-Learning environ-ments », Computers \& Electrical Engineering, vol. 87, p. 106791, oct. 2020, https://doi.org/10.1016/ j.compeleceng. 2020.106791 


\section{Authors}

Sonia Souabi, PhD student at Mohammadia School of Engineers in Mohammed V University of Rabat, Morocco, Asmaâ Retbi, Mohammed Khalidi Idrissi and Samir Bennani are professors at Mohammadia school of engineers, they work on recommendation systems within social learning networks in the context of e-learning. Their research team works on the application of IT tools and data analysis in the elearning context. They have published several papers in many international conferences. (RIME Team-Networking, Modelling and e-Learning Team- MASI Laboratory- Engineering.3S Research center).

Article submitted 2020-08-31. Resubmitted 2020-10-23. Final acceptance 2020-10-24. Final version published as submitted by the authors. 